\title{
A live, impaired-fidelity coronavirus vaccine protects in an aged, immunocompromised mouse model of lethal disease
}

\author{
Rachel L Graham ${ }^{1}$, Michelle M Becker ${ }^{2}$, Lance D Eckerle ${ }^{2}$, Meagan Bolles ${ }^{3}$, Mark R Denison ${ }^{2,4}$ \& \\ Ralph S Baric ${ }^{1,3}$
}

\begin{abstract}
Live, attenuated RNA virus vaccines are efficacious but subject to reversion to virulence. Among RNA viruses, replication fidelity is recognized as a key determinant of virulence and escape from antiviral therapy; increased fidelity is attenuating for some viruses. Coronavirus ( $\mathrm{CoV}$ ) replication fidelity is approximately 20 -fold greater than that of other RNA viruses and is mediated by a $3^{\prime} \rightarrow 5^{\prime}$ exonuclease (ExoN) activity that probably functions in RNA proofreading. In this study we demonstrate that engineered inactivation of severe acute respiratory syndrome (SARS)-CoV ExoN activity results in a stable mutator phenotype with profoundly decreased fidelity in vivo and attenuation of pathogenesis in young, aged and immunocompromised mice. The ExoN inactivation genotype and mutator phenotype are stable and do not revert to virulence, even after serial passage or long-term persistent infection in vivo. ExoN inactivation has potential for broad applications in the stable attenuation of CoVs and, perhaps, other RNA viruses.
\end{abstract}

Of the approximately 335 emerging infectious diseases that were identified between 1940 and 2004, 60.3\% originated in wildlife ${ }^{1}$. From past pandemics, it is clear that highly pathogenic zoonoses are major threats to global human health, economic stability and national security $^{1-4}$. SARS-CoV and swine influenza virus CA/04/09 H1N1 have caused substantial human morbidity and mortality in the 21 st century. Similar to influenza, CoVs have a strong history of host shifting and cross-species transmission ${ }^{5,6}$. In addition to the emergence of SARS$\mathrm{CoV}$ in 2002 , which caused $50 \%$ mortality in aged populations, several other human CoVs, such as HCoV-NL63, HCoV-OC43 and HCoV$229 \mathrm{E}$, probably emerged from animal reservoirs within the past 200 years $^{7,8}$. The sudden emergence of new respiratory viral pathogens from animals underscores the need for new, broadly applicable vaccine strategies that rapidly and rationally attenuate emerging zoonoses, especially to protect vulnerable populations in future outbreaks.

Vaccines have a long history of success in reducing viral disease burdens. Live, attenuated viruses are ideal vaccine candidates, as they elicit balanced innate and adaptive lifelong protective immune responses with low production and delivery costs 9 . Unfortunately, broadly applicable strategies for the rational design of live, attenuated virus vaccines have remained elusive, and vaccines attenuated by chemical treatment or passage can revert to virulence, resulting in disease outbreaks in unvaccinated and immunocompromised populations ${ }^{9}$. Moreover, the precise mechanism of attenuation often remains unclear; thus, the stability of the attenuation cannot be clearly evaluated or assured.
RNA viruses encode RNA-dependent RNA polymerases that lack efficient proofreading capabilities; the resulting high error rates, which range from $10^{-3}$ to $10^{-5}$ mutations per site per round of replication, render RNA viruses highly vulnerable to lethal mutagenesis using chemical agents ${ }^{10,11}$. High mutation rates generate considerable genomic diversity, allowing RNA viruses to rapidly adapt to changing environmental conditions and hosts ${ }^{12}$. Increased replication fidelity has been shown to reduce the virulence of poliovirus and chikungunya virus ${ }^{12-14}$ and has been proposed as a strategy for live, attenuated virus design ${ }^{15}$. CoVs encode the largest known RNA virus genomes $(26-32 \mathrm{~kb})$, exceeding the theoretical limits of viable RNA genome size ${ }^{11}$. Mutation rates are lower in CoVs than in other RNA viruses, approaching $2 \times 10^{-6}$ mutations per site per round of replication ${ }^{16}$. Nsp14, encoded in the viral replicase gene, contains a $3^{\prime} \rightarrow 5^{\prime}$ exoRNase (ExoN) of the DEDDh exonuclease superfamily ${ }^{17}$. In addition to the CoVs, ExoN homologs are present in the members of the Nidovirales order, whose genomes are $>20 \mathrm{~kb}$, but are not present in the smaller arteriviruses (with genomes of $12-16 \mathrm{~kb}$ ), suggesting that the ExoN had a crucial role in genome expansion ${ }^{16,18}$. In vitro, $3^{\prime} \rightarrow 5^{\prime}$ exoRNase activity has been demonstrated for recombinant SARS-CoV nsp14 (ref. 19). We have engineered and recovered viable ExoN inactivation mutants from mouse hepatitis virus (MHV-ExoN) and SARS-CoV (Urbani background, SARS-ExoN). Both MHV-ExoN and SARS-ExoN inactivations are maintained stably for more than ten passages

${ }^{1}$ Department of Epidemiology, University of North Carolina at Chapel Hill, Chapel Hill, North Carolina, USA. ${ }^{2}$ Department of Pediatrics, Vanderbilt University, Nashville, Tennessee, USA. ${ }^{3}$ Department of Microbiology and Immunology, University of North Carolina at Chapel Hill, Chapel Hill, North Carolina, USA. ${ }^{4}$ Department of Pathology, Microbiology and Immunology, Vanderbilt University, Nashville, Tennessee, USA. Correspondence should be addressed to R.S.B. (rbaric@email.unc.edu). 
in vitro and have 15 - to 20 -fold increased mutation frequencies compared to wild-type MHV and SARS-CoV ${ }^{16,20}$. Thus, ExoN has a crucial role in CoV RNA genome replication fidelity in vitro, probably by directly mediating or stimulating proofreading, a function previously unknown among RNA viruses ${ }^{21}$.

In this study, we used the stable, low-fidelity mutator phenotype of the SARS-CoV ExoN mutants to determine whether decreased replication fidelity could be used as a rational design strategy for a live, attenuated vaccine with broad potential applications to other viruses ${ }^{16,20,21}$. We evaluated (i) the impact of the inactivation of an RNA-proofreading exonuclease and the resultant mutator phenotype on $\mathrm{CoV}$ replication, fitness and pathogenesis; (ii) virus stability after passage or persistence in vivo; and (iii) the efficacy of using a decreased-fidelity mutant as a vaccine. Further, we assessed the potential for generating stably attenuated, reversion-resistant, immunogenic strains of known and newly identified CoVs to be used as vaccines in both immunocompetent and immunocompromised populations.

\section{RESULTS}

\section{The mutator phenotype and decreased fitness of MA-ExoN}

We engineered nsp14 ExoN inactivation mutations into the background of the virulent mouse-adapted SARS-CoV (MAwt), yielding MA-ExoN (Fig. 1a,b). We compared MAwt, which causes increased mortality and acute respiratory distress in young and aged mouse models ${ }^{22-24}$, and MA-ExoN in in vitro growth experiments (multiplicity of infection $(\mathrm{MOI})=0.1 \mathrm{PFU}$ per cell). MA-ExoN showed a stable growth defect of less than $1 \log$ (Fig. 1c). When placed in direct competition, MA-ExoN was clearly less fit than MAwt over successive rounds of infection (Supplementary Fig. 1a,b). At $6 \mathrm{~h}$ after infection (p.i.), MA-ExoN genome RNA levels were roughly equivalent to those of MAwt and were lower than those of MAwt at $12 \mathrm{~h}$ p.i.; by $24 \mathrm{~h}$ p.i., MA-ExoN genome RNA levels were approximately $10 \%$ of those of MAwt (Supplementary Fig. 1c). Thus, the data suggest that MAExoN is able to initiate and establish replication efficiently through times of peak RNA synthesis (0-6 h) but has impaired accumulation, which manifests late in one round of infection and is amplified over multiple rounds. These results are consistent with accumulating defects resulting from a markedly increased mutation rate (see the Discussion section).

Figure 1 The nsp14 ExoN mutator virus in a virulent mouse-adapted SARS-CoV isogenic background. (a) Genome organization, with the locations of the nsp14 coding sequence (black rectangle) and the mouseadapted mutations (triangles) shown. ORFla/b, ORFla and ORF1b. Structural proteins are labeled as follows: S, spike; E, envelope; M, membrane; N, nucleocapsid. (b) Nsp14 ExoN motifs, DEDD domain residues (underlined) and alanine substitutions (D90A and E92A) in motif I recovered in wild-type SARS-CoV and MAwt backgrounds. (c) Growth analysis (MOI = 0.1 PFU per cell) of wild-type SARS-CoV, S-ExoN, MAwt and MA-ExoN on Vero cells. Error bars, s.d. (d) Mutation frequency from complete genome sequencing of plaque isolates of MAwt and MA-ExoN ( $n=5$ for both) at passage 3. The increase in mean mutation frequency (horizontal lines) in MA-ExoN compared to MAwt $\left(11.5 \times\right.$ ) is indicated. ${ }^{*} P<0.01$ (Mann-Whitney nonparametric test for independent samples). (e) The mutations identified with complete genome sequencing across five clones from each group. Filled circles, nonsynonymous mutations; open circles, synonymous mutations; black, noncoding mutations; red, mutations present in more than one clone; blue, mutations present in only one clone. Mouse-adapted mutations are shown as triangles on the genome schematic and were present in all sequenced genomes.
We then sequenced RNA from multiple MAwt and MA-ExoN plaques. Both the MAwt background and engineered ExoN mutations were present in all sequenced MA-ExoN clones. Additionally, MA-ExoN accumulated 14-fold more unique mutations and had a mean 11.5-fold greater mutation frequency compared to MAwt $(P<0.01)$ (Fig. 1d,e). These results confirm that the growth and replication fidelity impairments of the nsp14 ExoN mutator phenotype are present in MA-ExoN and are indistinguishable from those in SARSExoN during replication in culture.

\section{MA-ExoN is attenuated in vivo}

To assess MA-ExoN virulence, we infected young (10-week-old) and aged (14-month-old) female BALB/c mice with MA-ExoN or MAwt (Fig. 2). Young mice infected with MAwt showed dose-dependent weight loss and recovery (Fig. 2a), though they had no observable dose-dependent differences in lung titers or clearance after day 4 p.i. (Fig. 2b). In contrast, young mice infected with MA-ExoN showed no signs of clinical disease and had high but not dose-dependent lung titers that were rapidly cleared by day 4 p.i. (Fig. 2a,b). We then compared MA-ExoN and MAwt infection in aged, immunosenescent mice ${ }^{25}$. Mice infected with either virus experienced dose-dependent weight loss (Fig. 2c); however, although lung titers were equivalent across all doses of MA-ExoN and MAwt on day 2 p.i., mice infected with MA-ExoN cleared the virus independent of inoculation dose, whereas mice infected with MAwt had begun to clear the virus from higher-titer infections more efficiently at day 4 p.i. than from lower-titer infections (Fig. 2d). Additionally, whereas aged mice infected with MA-ExoN had no mortality, MAwt-infected mice had dose-dependent mortality (Fig. 2e). As described previously ${ }^{23}$, we found little if any virus in other organs. These experiments demonstrate that MA-ExoN is attenuated in both young and aged diseased mice compared to virulent MAwt and that disease symptoms, when present, are less pronounced in MA-ExoN infections than MAwt infections.

A potential concern with live, attenuated vaccines is the chance that they could revert to virulence in vivo, particularly in immunocompromised individuals. Therefore, we assessed whether MA-ExoN was

a

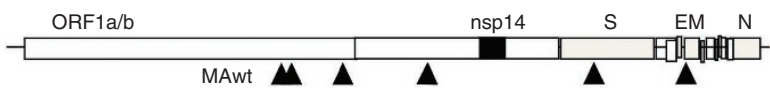

b

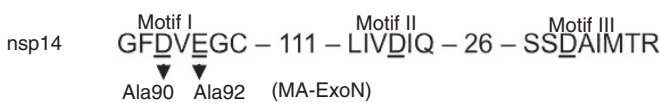

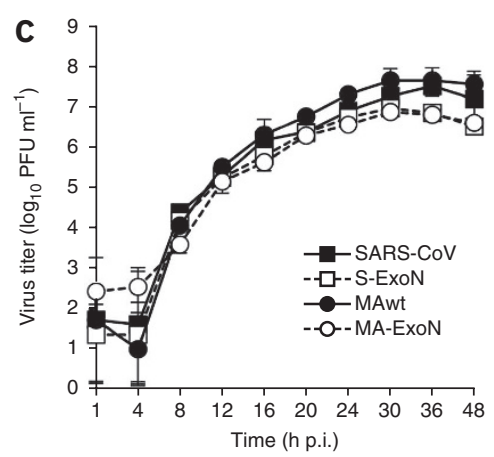

d

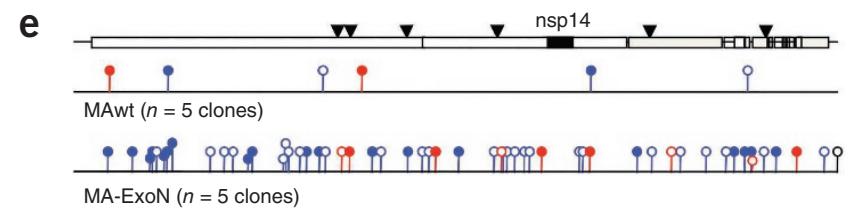



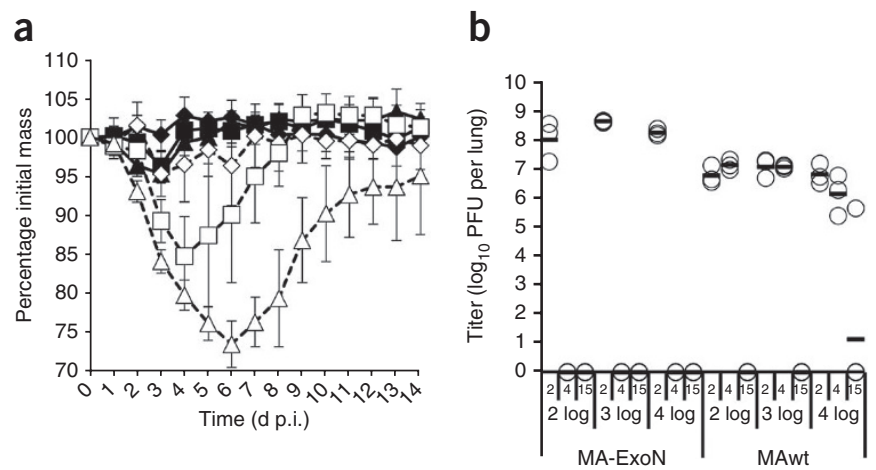

d

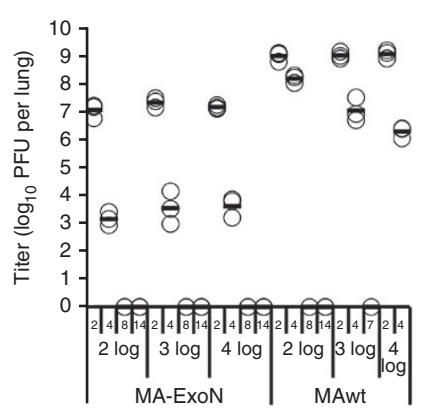

Figure 2 Weight loss and lung titer in BALB/c mice. (a-e) MA-ExoN and MAwt infections of 10-weekold $(\mathbf{a}, \mathbf{b})$ and 12- to 14-month-old (c-e) female BALB/c mice. (a,c) Weight loss. Dark shapes and solid lines, MA-ExoN; white shapes and dashed lines, MAwt; diamonds, $10^{2}$ PFU; squares, $10^{3}$ PFU; triangles, $10^{4}$ PFU. Error bars indicate s.d. (b,d) Lung titers. The titer for each mouse lung is indicated by an open circle; the mean titer of all mice at each time point is indicated by a horizontal bar. Days p.i. are indicated on the $x$ axes; $2 \log , 3 \log$ and $4 \log$ indicate the MOls of each virus $\left(10^{2}, 10^{3}\right.$ or $10^{4} \mathrm{PFU}$, respectively). (e) Survival within aged mouse groups, calculated as the percentage of surviving mice compared to the total number of mice remaining on each day of the experiment. Line weights and symbols are as in $\mathbf{a}$ and $\mathbf{c}$.

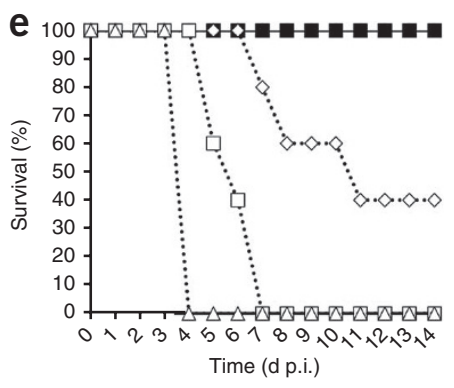

attenuated in immunocompromised mice. We used MAwt and MAExoN to infect young $\mathrm{Rag}^{-/-}$(recombination activating gene), severe combined immunodeficiency (SCID) and Stat ${ }^{-/-}$(signal transducer and activator of transcription 1) mice, as well as background controls (C57BL/6, BALB/c and 129 mice, respectively). All MA-ExoNinfected mice had significantly less weight loss than MAwt-infected mice $(P<0.05$; Fig. 3a-c and Supplementary Table 1$)$. Only Stat $1^{-/}$ mice had any notable weight loss $(\sim 15 \%)$ as a result of MA-ExoN infection; however, these mice did not pass experimental morbidity thresholds (Fig. 3c). In contrast, all MAwt-infected Stat $1^{-1-}$ mice died or were moribund by day 9 p.i., but MAwt infection was not lethal in C57BL/6 or 129 control mice (Fig. 3a,c), as has been previously
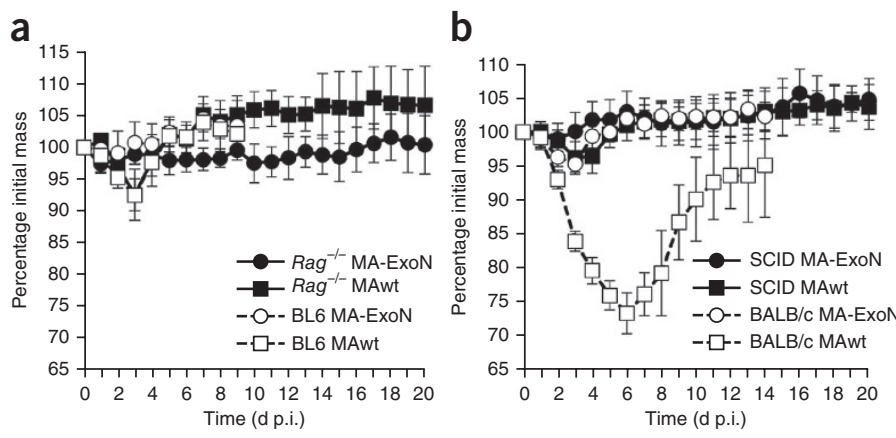

Figure 3 Weight loss and lung titer in young immunocompromised mice.

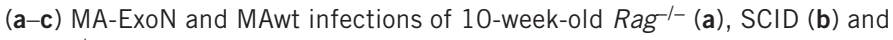
Stat1 ${ }^{-1-}$ (c) mice. C57BL/6 (a), BALB/c (b) and 129 (c) mice were included as background controls. Weight loss is shown. Dark shapes and solid lines, immunocompromised mice; white shapes and dashed lines, background control mice; circles, MA-ExoN; squares, MAwt. Error bars indicate s.d. (d-f) Lung titers for $\mathrm{Rag}^{-1-}$ (d), SCID (e) and Stat1--- (f) mice and background controls. The titer of each mouse lung is indicated by an open circle; the mean of all titers at each time point is indicated by a horizontal bar. Days p.i. are indicated on the $x$ axes.

reported ${ }^{22,26} \cdot \mathrm{Rag}^{-1-}$ and SCID mice maintained detectable amounts of MAwt and MA-ExoN virus for $14 \mathrm{~d}\left(\mathrm{Rag}^{-1-}\right)$ or $60 \mathrm{~d}$ (SCID) beyond the background controls (Fig. 3d,e) but showed no signs of illness over the course of the experiment despite a lack of viral clearance, expanding earlier reports from our laboratory that MAwt does not clear from $\mathrm{Rag}^{-1-}$ mice $^{26}$. The rapid clearance of MA-ExoN infection from Stat $^{-1-}$ mice (Fig. 3f) further supports the hypothesis that clearance of SARS-CoV infection is dependent on both $\mathrm{B}$ and $\mathrm{T}$ cells $\mathrm{s}^{27}$.

\section{Mutation accumulation during persistent in vivo infection}

Infection with both MAwt and MA-ExoN persisted for at least $60 \mathrm{~d}$ in SCID mice (Fig. 3e), potentially allowing for the most longitudinal
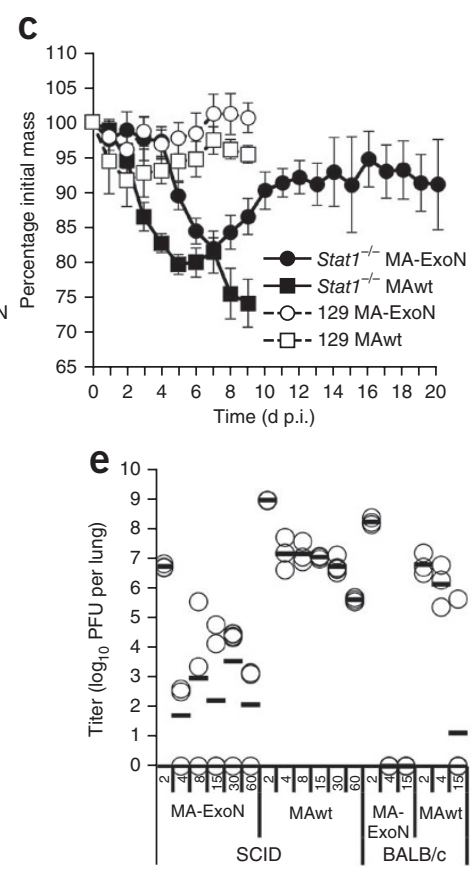

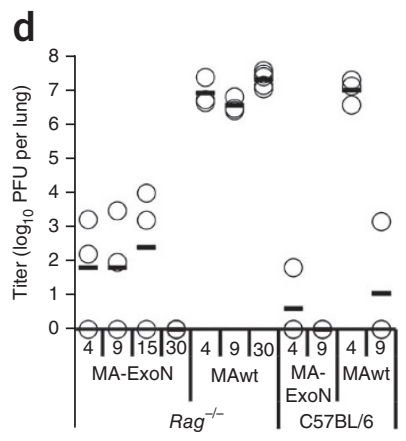

f

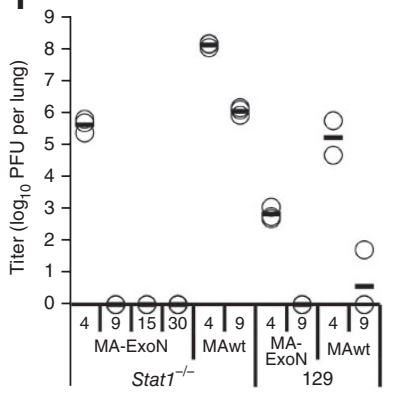


Figure 4 Mutation accumulation in infected SCID mice at $30 \mathrm{~d} \mathrm{p.i.}$ The SARS-CoV genome is depicted at the top. The nsp14 ExoN coding region is denoted by a purple box, with the inactivating amino acid changes indicated above the schematic. The receptor-binding domain (RBD) is denoted by a black box. Individual SCID mouse genome sequences are represented by black horizontal lines. Dashed lines separate the nonstructural protein sequences in ORF1 and downstream ORFs. Mutations are indicated by lollipop shapes colored as follows: blue, synonymous, unique to one sequence; light blue, synonymous, present in three sequences; red, nonsynonymous, unique to one sequence; green, synonymous, present in two sequences; purple, nsp14 ExoN inactivation mutations. Mutations that alter the size of an ORF are indicated by a red $\Delta$ (deletion) or a red S (stop codon). Genome sizes, ORF and nonstructural protein boundaries and mutation marker placements are approximate.

cycles of replication and lowest immune barriers to the emergence of mutations conferring increased fitness, reversion to virulence and fidelity-compensating changes of any of the groups examined. To test this, we sequenced viral genomes from viral plaques grown from SCID mouse lung homogenates at $30 \mathrm{~d}$ p.i. (Fig. 4 and Supplementary Table 2). We identified a total of 14 consensus mutations ( 100,000 nt) for MAwt, with 3 mutations shared in two or three genomes, resulting in 11 distinct mutations (4 synonymous and 7 nonsynonymous). For MA-ExoN, the engineered inactivation mutations were maintained. In contrast to MAwt, MA-ExoN plaques contained a total of 91 mutations (89 distinct: 32 synonymous and 57 nonsynonymous), corresponding to a 9.6-fold higher total mutation accumulation compared to MAwt.

We compared mutation accumulations across two separate regions (open reading frame 1a (ORF1a), nucleotides 493-8603, and ORF1b, nucleotides 12915-16520; Supplementary Figs. 2 and 3 and

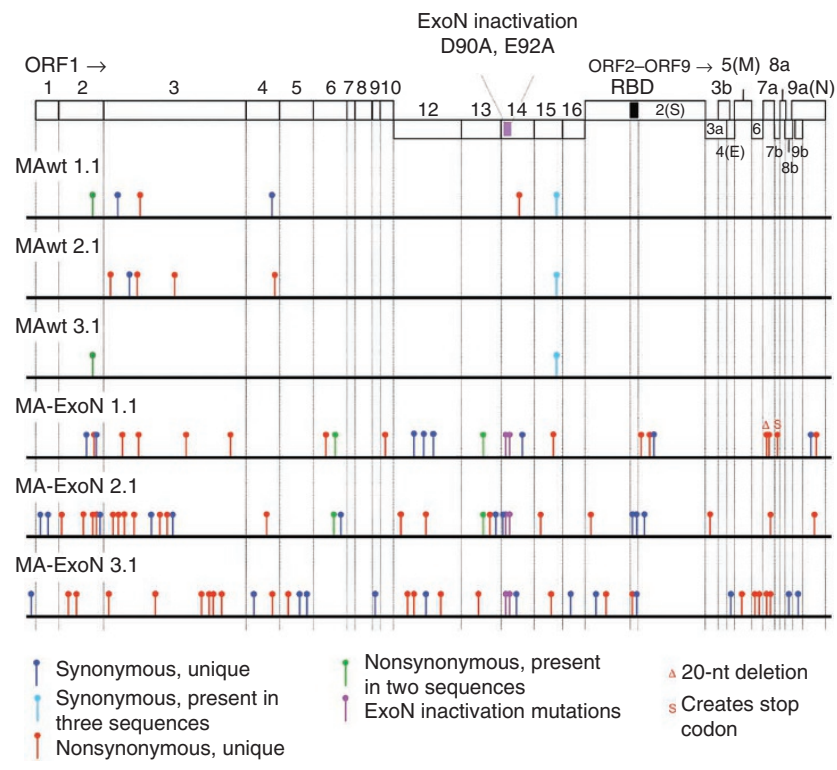

Supplementary Table 2) for statistical determinations. Mutation accumulations were significantly higher in MA-ExoN-infected mice for both regions $(P<0.01)$. Additionally, there was a mean 18.3 -fold increased accumulation for MA-ExoN across the ORF1a region. When we normalized the accumulations of mutations per $10 \mathrm{~kb}, \mathrm{MA}$ ExoN mutation accumulations in the ORF1a compared to the ORF1b region were not significantly different $(P=0.340)$ but remained significantly increased compared to the accumulation in MAwt $(P<0.001$ for both ORF1a and ORF1b). We identified no new
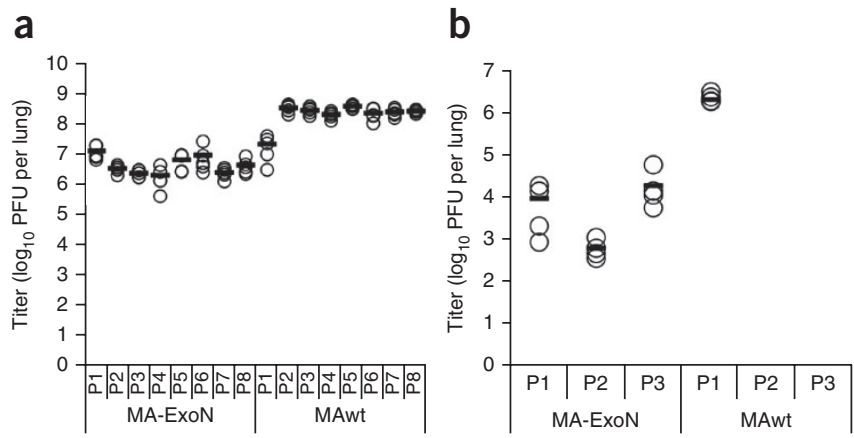

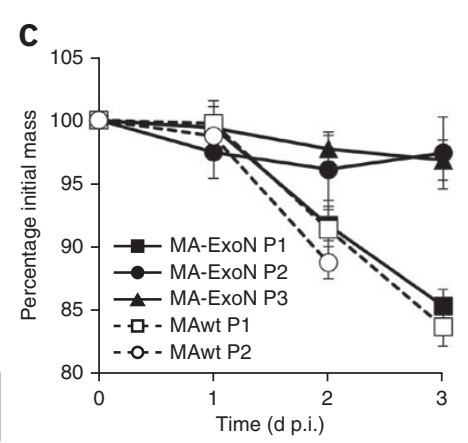

Figure 5 Virulence of passaged MA-ExoN and MAwt viruses. (a-c) MA-ExoN and MAwt serial infections of 12-month-old female BALB/c mice. Passages (P) are indicated on the $x$ axes. (a) Serial passage after 24 h. (b,c) Serial passage after $72 \mathrm{~h}$. $(\mathbf{a}, \mathbf{b})$ Lung titers are shown, with average titers indicated by bars. In the 72-h passage, MAwt-infected mice died by day 3 of passage 2; thus, the viruses were not passaged further. (c) Weight loss in the mice infected in the 72-h passage is shown. Error bars indicate s.d. (d) MA-ExoN (24-h passage 8 and 72-h passage 3 ) and MAwt (passage 1) infections of 12-month-old female BALB/c mice. Weight loss is shown. Error bars indicate s.d. (e) Infections of 10-week-old female BALB/c mice with MA-ExoN and MAwt population viruses isolated from SCID mice at $30 \mathrm{~d} \mathrm{p.i.;}$ weight loss is shown. Dashed line, mock (PBS) inoculation; dark shapes and solid lines, MA-ExoN; white shapes and solid lines, MAwt.

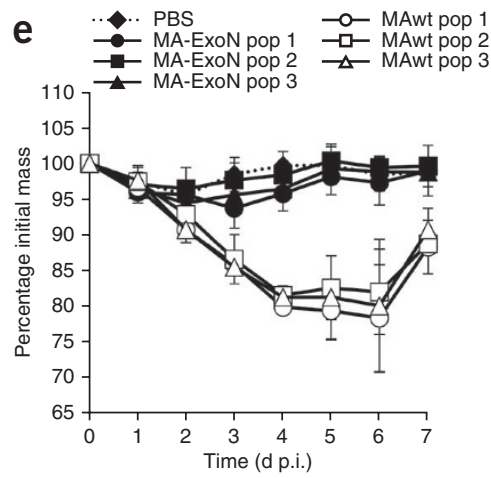

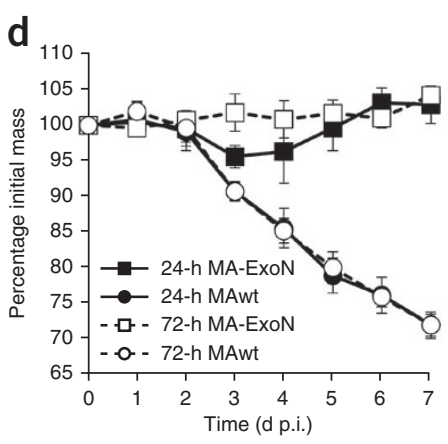

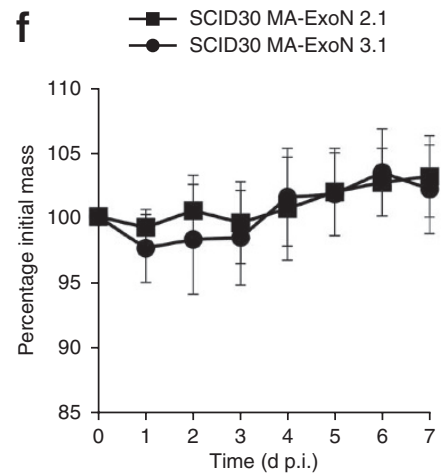

For each virus, lungs from three separate mice were harvested (mouse 1-mouse 3), and viruses were subsequently inoculated without plaque purification (population); circles, mouse 1 populations (pop 1); squares, mouse 2 populations (pop 2); triangles, mouse 3 populations (pop 3). (f) Weight loss in 11-month-old female BALB/c mice infected with MA-ExoN 2.1 (with C16999A) or MA-ExoN 3.1 (without C16999A) plaque isolates from SCID mice after $30 \mathrm{~d}$ of infection (SCID30). Squares, SCID30 MA-ExoN 2.1; circles, SCID30 MA-ExoN 3.1. Error bars indicate s.d. 
Figure 6 MA-ExoN vaccination protects from lethal challenge. $(\mathbf{a}, \mathbf{b})$ Low-passage MA-ExoN and mock (PBS) vaccinations of 12-month-old female BALB/c mice followed by lethal challenge with MAwt. (a) Weight loss in challenged mice. Dark circles, $10^{4}$ PFU MA-ExoN vaccination; white circles, $10^{2.5}$ PFU MA-ExoN vaccination; dark triangles, PBS vaccination. Error bars indicate s.d.

(b) Lung titers at day 2 after challenge. ExoN was given at a $2.5 \log \left(10^{2.5} \mathrm{PFU}\right)$ vaccination. The mean titer is indicated by a bar in each

group. (c) Fifty-percent plaque reduction neutralization titer (PRNT 50 ) assay using sera from PBS-vaccinated and MA-ExoN-vaccinated mice to neutralize MAwt. Reciprocal dilutions capable of effecting 50\% plaque reduction are shown by circles; mean reciprocal dilutions are indicated by a bar for each group. The limit of detection for each assay, if given, is indicated by a dashed line. mutations in any of the three MA-ExoN plaques, suggesting no obligatory or consistent pattern of adaptation or mutational bias. The most prevalent mutation identified $(\mathrm{C} 16999 \mathrm{M})$ was also present in viral stocks as a polymorphism; however, its frequency in the viral population remained stable ( $40 \%)$ both in vitro and in vivo and in experiments with both BALB/c and SCID mice (Supplementary Tables 2 and 3 and data not shown). The results from persistent ExoN infection over $30 \mathrm{~d}$ were consistent with the results from passage of SARSExoN virus in culture: the ratios of accumulation of unique mutations (MA-ExoN:MAwt) during replication in SCID mice ranged from $\sim 9.6: 1$ to $18.3: 1$, which is similar to the ratio measured between SARSExoN and SARS-CoV in culture ${ }^{16}$.

\section{MA-ExoN resists reversion to virulence in vivo}

To test the resistance to reversion to virulence during passage in vivo, we subjected MAwt and MA-ExoN to both short and long serial passages ( $24 \mathrm{~h}$ and $72 \mathrm{~h}$ per passage, respectively) in aged BALB/c mice (Fig. 5). In both groups, viral titer remained stable from passage to passage (Fig. 5a,b); additionally, viral plaque phenotypes were preserved, and the ExoN-inactivating mutations and amino acid substitutions were maintained (data not shown). In contrast, whereas MAwt titer remained stable over the 24 -h serial passage, mice inoculated with lung homogenates from the 72 -h passage died by day 3 in passage 2 (Fig. 5b). Although MA-ExoN titer remained stable over the 72 -h passage, mouse weights did not decrease during infection after passage 1 (Fig. 5c). Notably, there was no evidence of a gain of virulence with serial passage in the MA-ExoN pathogenesis model: when aged mice were infected with lung homogenates from each of the final 24-h and 72-h passages, mice infected with MA-ExoN passages lost no or little weight, and MAwt-infected mice became moribund (Fig. 5d).

To test whether persistent MA-ExoN infection could result in phenotypic reversion to virulence, we used viruses harvested from SCID mice at day 30 p.i. to infect young BALB/c mice (Fig. 5e). MAwtinfected mice showed signs of morbidity (weight loss, hunched posture and ruffled fur) but recovered. In contrast, MA-ExoN-infected mice showed no clinical signs of illness, as in the initial infection (Fig. 2a). Additionally, plaques containing different mutational subsets were identically attenuated after re-infection of BALB/c mice (Fig. 5f). These results demonstrate that after $30 \mathrm{~d}$ of persistent infection, the ExoN mutator phenotype did not revert to virulence despite the greatly increased mutation rate and population diversity.

\section{MA-ExoN vaccination protects mice from lethal challenge}

Aged mice mount poor productive immune responses to SARS-CoV vaccines and remain highly susceptible to severe disease and lethal infection $^{24,28}$, thus representing the most sensitive means to measure vaccine efficacy against lethal SARS-CoV infection. To test the efficacy of the MA-ExoN mutant as a possible vaccine against lethal challenge, we vaccinated aged BALB/c mice with MA-ExoN and allowed them to recover from infection. We then challenged the mice with a lethal dose of MAwt (Fig. 6). Mock-vaccinated mice succumbed to MAwt challenge by day 3 p.i. and had high lung titers (Fig. 6a,b); however, mice vaccinated with MA-ExoN were protected from illness (Fig. 6a). Further, in contrast to other vaccine platforms ${ }^{24,28,29}$, MA-ExoN-vaccinated mice had no detectable lung titers $2 \mathrm{~d}$ after challenge (Fig. 6b).

Additionally, mice vaccinated with MA-ExoN generated high amounts of neutralizing antibodies (mean, 1:311 \pm 37.5 reciprocal $50 \%$ neutralization titer) (Fig. 6c). The minimal neutralizing titers for protection against SARS-CoV infection in mice have been reported as 1:25-1:49 (ref. 30). Thus, even with a single vaccination, MA-ExoN provided complete protection against lethal challenge in a susceptible, immunosenescent mouse model of lethal SARS-CoV infection. To our knowledge, the MA-ExoN virus is the first approach to SARS-CoV immunization that fully protects against clinical disease and viral replication in an aged mouse model $24,28,29$.

\section{DISCUSSION}

Live, attenuated vaccines have substantially reduced the global disease burden associated with viral infections, including, for example, those of measles, mumps, rubella, polio, yellow fever and chickenpox ${ }^{9,15}$. However, live, attenuated vaccines carry several risks, including primary or secondary reversion to a virulent phenotype, as in the case of poliovirus ${ }^{9}$. In fact, attenuated phenotypes encounter natural selective pressures for reversion that can cause outbreaks of disease in unvaccinated populations ${ }^{31}$.

RNA virus replication fidelity has evolved to balance genome diversity and stability; therefore, inactivating an enzyme, such as nsp14-ExoN, that is responsible for high-fidelity replication could theoretically drive the virus toward instability, deleterious mutational diversity and decreased fitness in complex environments, which we observed here in vitro. It is possible that nsp14-ExoN may have other functions in viral RNA synthesis ${ }^{21}$; however, global impairment of viral RNA synthesis alone cannot explain the in vitro results and the attenuating phenotype in vivo.

It is not possible to fully separate defects caused by increased mutation load from those resulting from the replication defect observed, and both probably contribute to the phenotype. However, our results are consistent with the hypothesis that both stable and evolving defects resulting from the mutator phenotype have irrevocably attenuated MA-ExoN. These defects could include (i) mutations that impair or terminate translation, replication and transcription; 
(ii) mutations that impair or abolish protein functions; or (iii) changes in RNA polymerase processivity in the presence of an inactivated proofreading exonuclease. These combined impairments may be manifested with CoV ExoN mutants, as the high number of accumulated mutations per genome is unprecedented among viral mutator strains. Indeed, the phenotype observed with CoV ExoN inactivation is similar to those reported for other polymerase complexes with inactivated exonucleases, such as human mitochondrial DNA polymerase $\gamma(\text { pol } \gamma)^{32,33}$ and bacteriophage T7 DNA polymerase ${ }^{34-36}$. In pol $\gamma$ studies, the loss of proofreading was associated with impaired polymerase activity in a manner that was probably causal, impossible to uncouple and characterized by decreased speed, increased template dissociation and restricted access of nucleotides to the polymerase active site $^{32,33}$. For CoVs, the high levels of iterative amplification of both genomic and subgenomic RNA would further accelerate these deleterious processes by providing aberrant templates. The loss of ExoN proofreading would continuously generate new potentially attenuating alleles, and defective genomes and would reduce both genome fitness and the risks for primary and secondary reversions to virulence.

In this study, we demonstrate that MA-ExoN is attenuated in mice and that the mutant clears rapidly in the presence of an adaptive immune response. Although our in vivo experiments with MAwt recapitulated many of the phenotypes observed in aged and immunocompromised human populations, additional testing of MA-ExoN as a vaccine in primates will be necessary to further confirm its stability in vivo ${ }^{37}$. Experiments in SCID mice with persistent MA-ExoN infection verified the accumulation of mutations across the genome without evidence for the selection of either phenotypic virulence-enhancing alleles or primary genotypic reversion. Viruses harvested after passage remained avirulent, supporting the conclusions that (i) selection for virulence is not occurring; (ii) selection is being outcompeted by the gradual accumulation of attenuating mutations in individual genomes or the population mutational swarm; or (iii) the mutant is unable to generate or select for either fast-growing or slow-clearing viruses that are also more virulent. Not surprisingly, we identified a limited number of polymorphisms in the MA-ExoN virus stock that we used in subsequent experiments. Viruses with and without these mutations were fully attenuated in vivo, and the mutations were also maintained in lungs in the same frequencies as in the virus stock, suggesting that they were not selected against during passage in vivo. Thus, no single mutation or polymorphism could be clearly linked to viral attenuation except for the ExoN inactivation.

Notably, we have shown that MA-ExoN vaccinations are completely protective against replication and lethal challenge in aged BALB/c mice, the SARS-CoV mouse pathogenesis model that captures most of the severe clinical disease outcomes in human infections. Additionally, neutralization titers were equivalent or superior to those reported in studies of two-dose alphavirus replicon $S$ glycoprotein vaccines and killed vaccines containing alum, with the additional advantage of protecting against virus replication and clinical disease $\mathrm{e}^{24,28,29}$.

Live, attenuated vaccines must have two characteristics, aside from the capacity to elicit a protective immune response: resistance to primary reversion and stable attenuation at secondary sites. We have demonstrated that MA-ExoN has both of these characteristics. In all circumstances, the engineered inactivation mutations were maintained, indicating that exonuclease activity is not crucial for the virus life cycle and that the 4-nt, 2-amino acid change presents a substantial barrier to primary reversion; further, the passage experiments suggest that the virus lacks redundant or complementing mechanisms to fully restore the loss of ExoN activity. In addition, MA-ExoN harvested from persistently infected SCID mice retained an attenuated phenotype when re-inoculated into young $\mathrm{BALB} / \mathrm{c}$ mice, suggesting that persistence does not select for virulence. Future studies are necessary to address whether additional modifications could enhance and stabilize the attenuated phenotype by reducing the likelihood of gain of function by homologous recombination, such as introduction of the ExoN inactivation in a background with rewired transcriptional regulatory sequences ${ }^{38}$, which could increase resistance to reversion.

The inactivation of putative viral proofreading components in the pursuit of a stable vaccine constitutes a paradigm that has high potential to be broadly applicable to those members of the Nidovirales order with an exonuclease activity. In a time when metagenomics studies inform us of the likelihood of future viral emergence events-viruses that have the potential to afflict the human population much as SARSCoV did in 2002-2003-the design and ready implementation of an attenuation strategy that can be rapidly applied to any emerging $\mathrm{CoV}$ potentially represents a major advance in the preservation of public health. These data should also encourage the pursuit of fidelityimpairing mutations in the replicase proteins of other RNA viruses as potential targets or the use of CoVs as vaccine vectors for rational vaccine design.

\section{METHODS}

Methods and any associated references are available in the online version of the paper.

Accession codes. Plaque-purified MA-ExoN isolate sequences are deposited in GenBank under the following accession codes: FJ882942; FJ882943; FJ882945; FJ882948; FJ882951; FJ882952; FJ882953; FJ882957; FJ882958; FJ882959; FJ882961; FJ882962; HQ890526; HQ890527; HQ890528; HQ890529; HQ890530; HQ890531; HQ890532; HQ890533; HQ890534; HQ890535; HQ890536; HQ890537; HQ890538; HQ890539; HQ890540; HQ890541; HQ890542; HQ890543; HQ890544; HQ890545; HQ890546; JF292902; JF292903; JF292904; JF292905; JF292906; JF292907; JF292908; JF292909; JF292910; JF292911; JF292912; JF292913; JF292914; JF292915; JF292916; JF292917; JF292918; JF292919; and JF292920.

Note: Supplementary information is available in the online version of the paper.

\section{ACKNOWLEDGMENTS}

The authors thank R. Halpin, C. Town (US National Institutes of Health Microbial Genome Sequencing Contract HHSN272200900007C) and X. Lu for their assistance in sequencing in vitro isolates. This work was funded by US National Institutes of Health grants U54-AI057157 (SERCEB; R.S.B. and M.R.D.), AI075297 (R.S.B.) and 5F32AI080148 (R.L.G.)

\section{AUTHOR CONTRIBUTIONS}

R.L.G. designed and performed experiments, analyzed data, and wrote and edited the paper. M.M.B., L.D.E. and M.B. performed experiments, analyzed data and read the paper. M.R.D. and R.S.B. designed experiments, analyzed data, and wrote and edited the paper.

\section{COMPETING FINANCIAL INTERESTS}

The authors declare no competing financial interests.

Published online at http://www.nature.com/doifinder/10.1038/nm.2972.

Reprints and permissions information is available online at http://www.nature.com/ reprints/index.html.

1. Jones, K.E. et al. Global trends in emerging infectious diseases. Nature 451 , 990-993 (2008).

2. Li, Y. et al. On the origin of smallpox: correlating variola phylogenics with historical smallpox records. Proc. Natl. Acad. Sci. USA 104, 15787-15792 (2007).

3. Morens, D.M. \& Fauci, A.S. The 1918 influenza pandemic: insights for the 21 st century. J. Infect. Dis. 195, 1018-1028 (2007). 
4. Sessa, R., Palagiano, C., Scifoni, M.G., di Pietro, M. \& Del Piano, M. The major epidemic infections: a gift from the Old World to the New? Panminerva Med. 41, 78-84 (1999).

5. Cheng, V.C., Lau, S.K., Woo, P.C. \& Yuen, K.Y. Severe acute respiratory syndrome coronavirus as an agent of emerging and reemerging infection. Clin. Microbiol. Rev. 20, 660-694 (2007)

6. Graham, R.L. \& Baric, R.S. Recombination, reservoirs, and the modular spike: mechanisms of coronavirus cross-species transmission. J. Virol. 84, 3134-3146 (2010).

7. Pfefferle, S. et al. Distant relatives of severe acute respiratory syndrome coronavirus and close relatives of human coronavirus $229 \mathrm{E}$ in bats, Ghana. Emerg. Infect. Dis. 15, 1377-1384 (2009).

8. Vijgen, L. et al. Complete genomic sequence of human coronavirus OC43: molecular clock analysis suggests a relatively recent zoonotic coronavirus transmission event. J. Virol. 79, 1595-1604 (2005).

9. Vignuzzi, M., Wendt, E. \& Andino, R. Engineering attenuated virus vaccines by controlling replication fidelity. Nat. Med. 14, 154-161 (2008).

10. Drake, J.W. \& Holland, J.J. Mutation rates among RNA viruses. Proc. Natl. Acad. Sci. USA 96, 13910-13913 (1999).

11. Holland, J.J., Domingo, E., de la Torre, J.C. \& Steinhauer, D.A. Mutation frequencies at defined single codon sites in vesicular stomatitis virus and poliovirus can be increased only slightly by chemical mutagenesis. J. Virol. 64, 3960-3962 (1990).

12. Vignuzzi, M., Stone, J.K., Arnold, J.J., Cameron, C.E. \& Andino, R. Quasispecies diversity determines pathogenesis through cooperative interactions in a viral population. Nature 439, 344-348 (2006).

13. Coffey, L.L., Beeharry, Y., Borderia, A.V., Blanc, H. \& Vignuzzi, M. Arbovirus high fidelity variant loses fitness in mosquitoes and mice. Proc. Natl. Acad. Sci. USA 108, 16038-16043 (2011)

14. Lauring, A.S. \& Andino, R. Quasispecies theory and the behavior of RNA viruses. PLoS Pathog. 6, e1001005 (2010).

15. Lauring, A.S., Jones, J.O. \& Andino, R. Rationalizing the development of live attenuated virus vaccines. Nat. Biotechnol. 28, 573-579 (2010).

16. Eckerle, L.D. et al. Infidelity of SARS-CoV nsp14-exonuclease mutant virus replication is revealed by complete genome sequencing. PLoS Pathog. 6, e1000896 (2010).

17. Snijder, E.J. et al. Unique and conserved features of genome and proteome of SARS-CoV, an early split-off from the coronavirus group 2 lineage. J. Mol. Biol. 331, 991-1004 (2003).

18. Nga, P.T. et al. Discovery of the first insect nidovirus, a missing evolutionary link in the emergence of the largest RNA virus genomes. PLoS Pathog. 7, e1002215 (2011).

19. Minskaia, E. et al. Discovery of an RNA virus $3^{\prime} \rightarrow 5^{\prime}$ exoribonuclease that is critically involved in coronavirus RNA synthesis. Proc. Natl. Acad. Sci. USA 103, 5108-5113 (2006).

20. Eckerle, L.D., Lu, X., Sperry, S.M., Choi, L. \& Denison, M.R. High fidelity of murine hepatitis virus replication is decresed in nsp14 exoribonuclease mutants. J. Virol. 81, 12135-12144 (2007)
21. Denison, M.R., Graham, R.L., Donaldson, E.F., Eckerle, L.D. \& Baric, R.S Coronaviruses: an RNA proofreading machine regulates replication fidelity and diversity. RNA Biol. 8, 270-279 (2011).

22. Frieman, M.B. et al. SARS-CoV pathogenesis is regulated by a STAT1 dependent but a type I, II and III interferon receptor independent mechanism. PLoS Pathog. 6 , e1000849 (2010).

23. Roberts, A. et al. A mouse adapted SARS coronavirus causes disease and mortality in BALB/c mice. PLoS Pathog. 3, e5 (2007).

24. Sheahan, T. et al. Successful vaccination strategies that protect aged mice from lethal challenge from influenza virus and heterologous severe acute respiratory syndrome coronavirus. J. Virol. 85, 217-230 (2011).

25. Frieman, M. et al. Molecular determinants of severe acute respiratory syndrome coronavirus pathogenesis and virulence in young and aged mouse models of human disease. J. Virol. 86, 884-897 (2012).

26. Sheahan, T. et al. MyD88 is required for protection from lethal infection with a mouse-adapted SARS-CoV. PLoS Pathog. 4, e1000240 (2008).

27. Zhao, J., Van Rooijen, N. \& Perlman, S. Evasion by stealth: inefficient immune activation underlies poor T cell response and severe disease in SARS-CoV-infected mice. PLoS Pathog. 5, e1000636 (2009).

28. Bolles, M. et al. A double-inactivated severe acute respiratory syndrome coronavirus vaccine provides incomplete protection in mice and induces increased eosinophilic proinflammatory pulmonary response upon challenge. J. Virol. 85, 12201-12215 (2011).

29. Deming, D. et al. Vaccine efficacy in senescent mice challenged with recombinant SARS-CoV bearing epideic and zoonotic spike variants. PLoS Med. 3, e525 (2006).

30. Subbarao, K. et al. Prior infection and passive transfer of neutralizing antibody prevent repication of SARS coronavirus in the respiratory tract of mice. J. Virol. 78 3572-3577 (2004).

31. Kew, O. et al. Outbreak of poliomyelitis in Hispaniola associated with circulating type 1 vaccine-derived poliovirus. Science 296, 356-359 (2002).

32. Johnson, A.A. \& Johnson, K.A. Exonuclease proofreading by human mitochondrial DNA polymerase. J. Biol. Chem. 276, 38097-38107 (2001).

33. Johnson, A.A. \& Johnson, K.A. Fidelity of nucleotide incorporation by human mitochondrial DNA polymerase. J. Biol. Chem. 276, 38090-38096 (2001).

34. Donlin, M.J., Patel, S.S. \& Johnson, K.A. Kinetic partitioning between the exonuclease and polymerase sites in DNA error correction. Biochemistry 30 538-546 (1991).

35. Patel, S.S., Wong, I. \& Johnson, K.A. Pre-steady-state kinetic analysis of processive DNA replication including complete characterization of an exonuclease-deficient mutant. Biochemistry 30, 511-525 (1991).

36. Wong, I., Patel, S.S. \& Johnson, K.A. An induced-fit kinetic mechanism for DNA replication fidelity: direct measurement by single-turnover kinetics. Biochemistry 30 526-537 (1991).

37. Smits, S.L. et al. Exacerbated innate host response to SARS-CoV in aged non-human primates. PLoS Pathog. 6, e1000756 (2010)

38. Yount, B., Roberts, R.S., Lindesmith, L. \& Baric, R.S. Rewiring the SARS-CoV transcription circuit: engineering a recombination-resistant genome. Proc. Natl. Acad. Sci. USA 103, 12546-12551 (2006). 


\section{ONLINE METHODS}

Construction of SARS D plasmid with ExoN and mouse-adapted mutations. A SARS-CoV D plasmid was constructed by restriction digestion and ligation of existing SARS D-ExoNI ${ }^{16}$ and SARS D mouse-adapted ${ }^{23}$ plasmids. Briefly, both plasmids were restriction digested with BstB I and Xba I enzymes. After treatment of the digested SARS D-ExoNI plasmid with Antarctic Phosphatase (New England BioLabs, Ipswitch, MA, USA), fragments were isolated, purified and ligated together using T4 DNA Ligase overnight at $4{ }^{\circ} \mathrm{C}$ as described previously ${ }^{39}$. Colonies were screened for proper insert size by restriction digestion and electrophoresis, and the presence of the appropriate mutations was verified by sequencing.

Generation of SARS-CoV MA-ExoN mutant virus. Virus containing the ExoNI inactivation and mouse-adapted mutations within the viral coding sequence was produced using the infectious complementary DNA (cDNA) assembly strategy for SARS-CoV as previously described ${ }^{39,40}$. ExoN viruses were kept at low passage (one passage past virus rescue, $\mathrm{P} 1$ ) to minimize the accumulation of mutations in cell culture. For this study, an equivalently low-passaged MAwt virus was used for comparison.

In vitro passage series and viral growth and plaque assays. MAwt and MA-ExoN viruses were grown in Vero cells at MOI $=0.1 \mathrm{PFU}$ per cell in all in vitro experiments, with the exception of the genome RNA quantification experiment, which was performed at MOI $=3 \mathrm{PFU}$ per cell. Passage series and growth experiments were performed, and viral titers were determined as previously described ${ }^{16}$.

Competition assay. Vero cells were plated at $10^{6}$ cells per well in six-well plates. Cells were infected at an MOI of 0.1 with combinations of MAwt and MA-ExoN at 1:1, 1:10 and 1:100 ratios favoring either MA-ExoN or MAwt and were incubated for $24 \mathrm{~h}$ at $37^{\circ} \mathrm{C}$. After $24 \mathrm{~h}, 100-\mu \mathrm{l}$ aliquots of each supernatant were passed to fresh six-well plates of Vero cells for five total successive passages, and infected monolayers were harvested in TRIzol (Invitrogen, Grand Island, NY, USA). After passages were complete, RNA was purified according to the manufacturer's protocol, and first-strand cDNA was generated as described ${ }^{41}$. PCR products were produced using the primers S32F and S34R (Supplementary Table 4). Once the presence of single-band PCR products was verified by agarose gel electrophoresis and the yields were calculated by spectrometry, $100 \mathrm{ng}$ of each product was restriction digested using BsrF I, which cuts in the nsp14ExoN engineered mutation site but not in the corresponding MAwt sequence. Digested products were resolved on a 1.7\% agarose gel, and normalized relative percentages of MAwt and ExoN-MA digested products were calculated using ImageJ (http://rsbweb.nih.gov/ij/).

Quantification of genome RNA. Vero cells were infected with either MAwt or MA-ExoN at MOI $=3$ PFU per cell. At 6, 12 and $24 \mathrm{~h}$ p.i., RNA was harvested in TRIzol and isolated according to the manufacturer's protocol. First-strand cDNA was generated as described above, and real-time PCR was performed assessing for genome RNA using the primers 5 -AGCCAACCAACCTCGATCT CTTGT-3' (forward) and 5'-TGACACCAAGAACAAGGCTCTCCA-3' (reverse). cDNA was normalized using the GAPDH primers $5^{\prime}$-TGCACCACCAA CTGCTTAGC- $3^{\prime}$ (forward) and $5^{\prime}$-GGCATGGACTGTGGTCATGAG-3' $(\text { reverse })^{42}$. Normalized results were then compared as ratios of MA-ExoN to MAwt genomes using the $\Delta \Delta \mathrm{Ct}$ method.

Infection of mice with SARS-CoV MAwt and MA-ExoN. All experimental protocols involving mice were reviewed and approved by the institutional animal care and use committee at the University of North Carolina, Chapel Hill. The following groups of mice were used: 10-week-old female BALB/c (Charles River Laboratories, Wilmington, MA, USA), 14-month-old female BALB/c (Harlan Laboratories, Indianapolis, IN, USA), 10-week-old female Stat1 $1^{-/-}$ (Taconic Farms, Hudson, NY, USA; stock 002045-M-F), 10-week-old female 129S6/SvEvTac (Taconic; stock 129SVE-F), 10-week-old female Rag $^{-1-}$ (Jackson Labs, Bar Harbor, ME, USA; stock 002216), 10-week-old female C57BL/6
(Jackson; stock 00064) and 10-week-old female SCID (Jackson; stock 001803). Mice were lightly anesthetized and infected intranasally with varying doses $\left(10^{2}-10^{4} \mathrm{PFU}\right.$, depending on the experiment) of SARS-CoV MAwt or SARSCoV MA-ExoN. Mice were weighed daily, and on certain days specified in each experiment, a subset of mice in each group was euthanized, and their lungs were harvested for virus titer. Mice that dropped below $70 \%$ of their initial mass or were moribund were euthanized before their scheduled time points. Serial passages were inoculated as above for passage 1 ; subsequent passages were inoculated with $50 \mu \mathrm{l}$ of clarified lung homogenate (lungs were homogenized in $1 \mathrm{ml}$ of PBS) from the previous passage. All experiments used $n=5$ mice per virus per dosage per condition (if applicable) per time point, with the exception of experiments using immunocompromised mice, in which $n=3$.

Determination of virus titer in infected mouse lungs. Lungs harvested for virus titer were weighed and homogenized in $1.0 \mathrm{ml}$ PBS at 6,000 r.p.m. for $60 \mathrm{~s}$ in a MagnaLyser (Roche, Basel, Switzerland). Virus titers were determined by plaque assay on Vero cells as previously described ${ }^{39}$.

Determination of viral neutralization antibody titers in mouse sera. Mouse sera were heat inactivated for $30 \mathrm{~min}$ at $55^{\circ} \mathrm{C}$ and then serially diluted to $1: 100$, $1: 200,1: 400,1: 800$ and 1:1,600 in PBS to a volume of $125 \mu \mathrm{l}$. Next, $125 \mu \mathrm{l}$ of PBS containing low-concentration MAwt (40 PFU) or high-concentration MAwt (240 PFU) was added to each serum dilution. The virus-serum mixtures were incubated at $37^{\circ} \mathrm{C}$ for $30 \mathrm{~min}$. After incubation, virus titers of the mixtures were determined by plaque assay as described ${ }^{39}$. We then calculated the $\mathrm{PRNT}_{50}$ values, the serum dilutions at which plaque formation was reduced by $50 \%$ relative to that of virus stock not treated with serum.

Viral genome sequencing. To determine the sequences of viral genomes present in SCID mouse lungs after $30 \mathrm{~d}$ of infection, plaques were isolated from lung samples from SCID mice at $30 \mathrm{~d}$ p.i. as described above. Briefly, once individual, well-resolved viral plaques were visible, they were harvested by collecting the agarose plugs above them using a $200-\mu \mathrm{l}$ pipette tip. Each agarose plug was dropped in $0.5 \mathrm{ml} \mathrm{PBS}$, allowed to diffuse for $24 \mathrm{~h}$ at $4{ }^{\circ} \mathrm{C}$ and then applied to $\sim 70 \%$ confluent monolayers of Vero cells in T25 flasks and incubated for $48 \mathrm{~h}$ at $37^{\circ} \mathrm{C}$. Infected cell monolayers were then harvested in $1 \mathrm{ml}$ TRIzol. Firststrand cDNA was generated as described ${ }^{41}$. Amplicons of the viral genomes were generated as follows: for whole-genome sequencing (amplicons 1-13) and partial-genome sequencing (amplicons $A-G, X$ and $Y$ ), the primer pairs indicated in Supplementary Table 4 were used in a 50- $\mu$ PCR reaction using Phusion polymerase (New England BioLabs). Five microliters of each PCR reaction were electrophoresed on agarose gels to verify the presence of correctly sized amplicons, and PCR products were purified using a Qiagen PCR Purification Kit (Qiagen, Valencia, CA, USA). Amplicons were then sequenced using the corresponding primer sets for each amplicon, as indicated in Supplementary Table 4. Sequence results were analyzed using Geneious Pro 5.3.6 (Biomatters, Auckland, New Zealand) and Serial Cloner 2.1 (SerialBasics, http://serialbasics. free.fr/Home/Home.html).

Statistical analyses. Statistical analyses were performed using the MannWhitney $U$ test (http://elegans.som.vcu.edu/ leon/stats/utest.html). Significance was set at $P<0.05$.

39. Yount, B. et al. Reverse genetics with a full length infectious cDNA of the severe acute respiratory syndrome coronavirus. Proc. Natl. Acad. Sci. USA 100, 12995-13000 (2003).

40. Graham, R.L., Sims, A.C., Brockway, S.M., Baric, R.S. \& Denison, M.R. The nsp2 replicase proteins of murine hepatitis virus and severe acute respiratory syndrome coronavirus are dispensable for viral replication. J. Virol. 79, 13399-13411 (2005).

41. Becker, M.M. et al. Synthetic recombinant bat SARS-like coronavirus is infectious in cultured cells and in mice. Proc. Natl. Acad. Sci. USA 105, 19944-19949 (2008).

42. Chey, S., Claus, C. \& Liebert, U.G. Validation and application of normalization factors for gene expression studies in rubella virus-infected cell lines with quantitative real-time PCR. J. Cell. Biochem. 110, 118-128 (2010). 